\title{
Molecular Dynamics Study of Lithium Intercalation into -OH Functionalized Carbon Nanotube Bundle
}

\section{Bin Zheng ( $\nabla$ zhengbin@hfnu.edu.cn )}

Hefei Normal University

\section{Huaze Dong}

Hefei Normal University

Jinmiao Zhu

Hefei Normal University

\section{Yanping Wang}

Hefei Normal University

\section{Research Article}

Keywords:

Posted Date: February 25th, 2022

DOI: https://doi.org/10.21203/rs.3.rs-1326366/v1

License: (9) This work is licensed under a Creative Commons Attribution 4.0 International License. Read Full License 


\section{Abstract}

The influence of hydroxyl group (-OH) on carbon nanotube (CNT) interacting with lithium ions have been investigated via ab initio molecular dynamic (MD) simulations. Compared with the pure CNT, a higher efficiency has been observed for lithium ions intercalating into CNT-OH bundle. At lower lithium ions density and CNT bundle density, CNT-OH exhibits a higher intercalation efficiency than the pristine and ammonium functionalized CNTs. As the increasing of lithium ions densities and CNT bundle densities, lithium ions tend to intercalate into the interlayer between $\mathrm{CNT}-\mathrm{OH}$ tubes instead of the interior of $\mathrm{CNT}-\mathrm{OH}$ tubes. We also observe the destruction of hydroxyl groups during the intercalation of lithium ions into interlayer of $\mathrm{CNT}-\mathrm{OH}$ bundle. It is therefore suggested that eliminating the intercalation of lithium ions into interlayer between tubes is important for the design of lithium ion batteries.

\section{Introduction}

Since traditional fuel energy is limited and overexploited, the burst of global warming urged scientists to find the renewable or new kinds of energy forms. ${ }^{1}$ As for the applications of portable devices or vehicles, materials with excellent charging and recharging performances are in increasing demand. Among them, lithium ion batteries (LIBs) with advantages of excellent cyclic stability, high capacity density and good energy storage have shown tremendous application fields in daily-life. As the performances of energy transfer rely on materials adopted, utilization of nanotechnology to construct tunable and designable nano-materials with desirable characters becomes more and more important.

Due to the atomic structure and surface modification characters, carbon based nano-materials have attracted much attention for energy conversion and storage. Since their findings in early 1990s, carbon nanotubes (CNTs) have been recommended in diverse applications. ${ }^{2-3}$ They have many fascinating performances such as excellent electronic sensitivity, good mechanical strength, high surface area to volume ratio, good electronic sensitivities, high flexibility and extraordinary electro-catalytic activities which endow them with practical applications in sensors, actuators, energy storage equipment, et. al. ${ }^{4-5}$

Although significant progress has been made, the problem of solubility hampers further applications. Pristine CNTs are insoluble in water or organic solvents, and have strong tendencies to aggregate into bundles owing to the strong $\pi$-bonds on CNTs' surfaces. These strong covalent bonds lead to heterogeneous distribution and limit the application of CNTs in industrial scale. To fabricate efficient electrode, CNTs are treated either by covalent modification of chemical groups or non-covalently attachment of functional molecules. ${ }^{6-7}$ For electro-catalytic application, CNTs should be pre-treated before usage. The air oxidation ${ }^{8}$ and mixed acid $^{9}$ methods have been applied to confer CNT with oxygencontaining groups such as carboxylic or hydroxyl groups on the surface ${ }^{10}$ with which the exfoliation of bundles will be alleviated and enhance homogeneous dispersion. For pure CNTs, the simultaneous satisfaction of high energy density and power density is difficult to realize since the storage mechanism depends on a physical intercalation process. The doping as well as modification have been applied and obtained excellent outcomes. ${ }^{11-12}$ It was reported that the defected CNTs could attain $1116 \mathrm{~mA} \mathrm{~h} / \mathrm{g}$ in 
stoichiometric $\mathrm{LiC}_{2}{ }^{13-15}$, and the lithium insertion for CNTs can cover a range of 220 to $780 \mathrm{~mA} \mathrm{~h} / \mathrm{g}{ }^{16}$ Except for the solubility, high columbic efficiency is another key problem. Some research concentrated on the morphological modulations such as shape, porosity, diameter and thickness of CNTs. ${ }^{17-21}$ The mechanism of charge transfer between Li and deformed CNTs are also in great concern. It was found that Li intercalation may lead to CNTs deformation ${ }^{18}$ and surface adsorption mechanism was suggested for the process ${ }^{19}$.

To solve this simple yet key problem, studies should be conducted. Experiments are direct but time consuming and expensive. Simulation can thus be an alternative, guiding and complementary way. The first principle method showed that in $1 \mathrm{M} \mathrm{LiClO}_{4} \mathrm{EC} / \mathrm{DEC}$, the $\mathrm{Li}$ intercalation occurred at the closed ends exhibiting a reversible capacity of $125 \mathrm{~mA} \mathrm{~h} / \mathrm{g}^{20}$. Utilizing DFT calculations, the diffusion of Li in CNT armchair $(5,5)$ from radial and axial directions was reported ${ }^{21}$ and the lithium insertion capacities were reported to be dependent on chirality ${ }^{22}$. The influence of chiralities on Li interaction with CNTs was also calculated based on $\mathrm{DFT}^{23-24}$. It was shown that $\mathrm{Li}$ could quickly penetrate into CNTs or exist between neighboring CNTs. Defects in the side walls of CNTs presented easy diffusion of $\mathrm{Li}$ ions ${ }^{25-26}$. Ab initio study was used to study Li adsorption into CNTs with amine and carboxyl groups $(\mathrm{NH} 2 /((8,0))$ and $\mathrm{COOH} /((8,0))$, and shows more preferential adsorption tendencies compared with pristine CNTs. ${ }^{27}$ Different functional groups onto CNTs have been studied and it was inferred that the introduction of functional groups would induce the transition from semi-metal to semiconductor status. ${ }^{28}$ The enhanced intercalation of lithium ions into CNTs by amine functionalization was also studied using ab initio method. ${ }^{29}$

Although several outcomes were achieved, the information is still limited. All the conclusions from simulations were drawn with static states while the actual situations are dynamic processes. Therefore, the evaluation during a dynamic process is in great need. To evaluate the potential application of functional groups on lithium ion adsorption in kinetic, in this work we investigated the hydroxyl group functionalized CNTs from axial and radial directions with different lithium ions densities and CNTs bundles densities.

\section{Computational Methods}

Single walled CNTs (SWCNT) with chirality $(8,0)$ and saturated hydrogen atoms on each ends were constructed in this work. They have zigzag conformations with 128 carbon atoms in total. Seven repeat units were arrayed along the tube axis and the size was set at lengths of $17.84 \AA$ and diameters of $3.19 \AA$. The hydroxyl group functionalized CNTs (CNTs-OH) were formed by chemisorbing 24 hydroxyl groups at the side walls of CNT and four hydroxyl groups were averagely distributed at two ends. The origin of the coordinate system was set the same as pristine CNTs and CNT-NH ${ }_{2}$ located at the center of the nanotube referred to our previous work. ${ }^{29}$ The ends of the CNTs were initially set at $z=8.92 \AA$ and $-8.92 \AA$. Two different lithium ion densities were studied for lithium ions intercalation utilizing MD simulations. 
For low lithium density study, eight Li ions were set at the upper and bottom ends of CNTs. Their coordinates were set at $\left(0.85,1.47, z_{0}\right),\left(0.85,1.47, z_{0}\right),\left(5.20,3.00, z_{0}\right)$ and $\left(5.20,3.00, z_{0}\right)$, where $z_{0}=13.0$ or -13.0 $\AA$. To exclude the influence of Li ion with CNTs' interactions at the beginning of simulation, the distance between lithium and CNT end edge was set to be $4.1 \AA$. For high lithium ion density study, twenty lithium atoms were added into the system. Aside from previous eight lithium ions, additional twelve ions were set at $\left(3.90,2.25, z_{0}\right),\left(3.90,2.25, z_{0}\right),\left(6.06,3.50, z_{0}\right),\left(6.06,3.50, z_{0}\right),\left(5.00,0.00, z_{0}\right)$, $\left(0.00,5.00, z_{0}\right)$, where $z_{0}=13.0$ or $-13.0 \AA$. To make the study more specific, two confined hexagonal boxes with dimensions of $13.0 \times 13.0 \times 32.0 \AA^{3}$ and $15.0 \times 15.0 \times 32.0 \AA^{3}$ were adopted for the distance investigations of CNT-OH interacting with lithium ions.

$\mathrm{VMD}^{32}$ was utilized to create the initial structure of $\mathrm{CNT}-\mathrm{OH}$. The initial conformation with or without the addition of lithium ions were submitted for further calculations using Car-Parrinello MD simulations. In this work, Car-Parrinello MD $^{31}$ was performed using the CPMD software package ${ }^{30}$. In all calculations, Perdew, Burke, and Ernzerhof (PBE) exchange-correlation functional ${ }^{33-34}$ was used. To assure energy convergence, a plane wave set with 80 Ry kinetic energy cutoff was adopted for valence electrons. The Troullier-Martins normconserving nonlocal pseudopotential ${ }^{35}$ was utilized to describe the valence electrons and ionic cores interactions in the Kleinman-Bylander form. ${ }^{36}$ The preconditioned conjugategradients (PCG) method and the L-BFGS method ${ }^{37}$ were utilized for wave function optimization and geometry calculation. In the MD calculations, the temperature was set to $300 \mathrm{~K}$, the massive NoseHoover chain algorithm ${ }^{37-38}$ were utilized to achieve thermostatting. All the hydrogen atoms were substituted by deuterium atoms, and a time step of 4.0 a.u. $(0.09676 \mathrm{fs})$ and a fictitious electronic mass of 500 a.u were used. At the beginning of MD simulations, the electronic wave functions were quenched to the Born-Oppenheimer surface. The kinetics study lasted 20 ps for CNT-OH system, and trajectories were collected every five steps. Periodic boundary conditions (PBC) are applied in all directions to simulate a bundle of CNTs, and only the $₫$ point of the Brillouin zone was considered for periodically repeated system calculation.

\section{Results And Discussion}

The stability of CNT-OH was first determined by geometry optimization calculations. As calculated in our previous work ${ }^{29}$, the $\mathrm{C}-\mathrm{C}$ bond distance in pristine $\mathrm{CNT}$ is $1.42 \AA$ while for $\mathrm{CNT}-\mathrm{OH}$, a small distortion induced by the polar groups, leading to bond lengths of $1.52 \AA$. The bond length of the $\mathrm{C}-\mathrm{O}$ bond is 1.47 $\AA$. The starting conformation of lithium ions with CNTs was shown in Fig. 1. 20 ps simulations of CNT$\mathrm{OHs}$ with $\mathrm{Li}$ ions were performed using $\mathrm{Ab}$ initio MD simulations.

\section{Lithium ions intercalation into CNT bundles at low Li ion density}

We first simulated the system with a low Li ion density. A low bundle density with a hexagonal box with dimensions of $15.0 \times 15.0 \times 32.0 \AA^{3}$ is used, and eight Li ions were placed at the ends of CNT-OH bundle, as 
shown in Fig. 1 (c). Z coordinate denotes the height of the CNT-OH, whose center is set to zero. The two dashed lines represent the upper and lower boundaries of CNT-OH. As shown in Fig. $2(\mathrm{~h})$, half of the eight lithium ions first distribute evenly at the upper side and the lower side. Then they sequentially diffuse into the interior or the interstitial channel of CNT-OH from the upper part of the $\mathrm{CNT}-\mathrm{OH}$. It is shown that all ions locates in the range from $-8.9 \AA$ to $8.9 \AA$, indicating the successful intercalations of lithium ions into $\mathrm{CNT}-\mathrm{OH}$ bundle. The monitoring of $\mathrm{Z}$ for each $\mathrm{Li}$ ions indicates that the fastest $\mathrm{Li}$ ion enters the CNT-OH tube at 3ps, and the slowest one at 8 ps. As shown in Fig. 2 (f), the distribution of ions in Z suggest that the most of the Li ions locates at the upper part of the bundle, and only one locate at lower part. More importantly, the sharp peaks in the distribution profile suggest that the adhesions of Li ions onto CNT-OH tube are stable and cannot be easily broken at room temperature.

The radial distribution of lithium ions on the x-y axis and snapshot are shown in Fig. 2 (d) and (c), respectively. As presented in Fig. 2 (a), seven of the total eight Li ions enter into the CNT-OH interior, and one moves into the channel between neighboring $\mathrm{CNT}-\mathrm{OH}$ tubes. The lithium ions stay on a cylindrical surface with a radius of $1.4 \AA$ and distribute almost evenly within CNT-OH. Detailed inspections into the behaviors of the lithium ion in the interstitial channel process are shown in Fig. 2 (e) and (g). The only one lithium ion in the channel area first diffuses among tubes, and then connects with the surface hydroxyl group, and finally leads to the destruction of hydroxyl groups from the $\mathrm{CNT}-\mathrm{OH}$. This process is spontaneous and happens within 6-9 ps. A side view of system after lithium ion intercalation is presented in Fig. 2 (b).

To further validate the impact of lithium ions on the stabilities of hydroxyl groups, a hexagonal box with dimensions of $13.0 \times 13.0 \times 32.0 \AA^{3}$ was constructed. The smaller box dimensions give closer distances between CNT bundles. As presented in Fig. 3 (a), six of the total eight Li ions enter into the CNT-OH interior, and two move into the channel between neighboring CNT-OH tubes. The vivid image for lithium ions contribution in axial direction is shown in Fig. 3 (c). The MD simulations show that six lithium ions diffuse into the CNT interior and presents a cylindrical distribution along the central axis and presents a sharp distribution alongside the axis as shown in Fig. 3 (d) and (f). The left two lithium ions moves into the bundle interlayers with distances of 2.1 and $3.7 \AA$ to the CNT sidewalls. The snapshot of the interlayer situation is shown in Fig. 3 (e), and a side view of system after lithium ion intercalation is presented in Fig. 3 (b). Careful examinations of the behaviors of lithium ion locating in the interlayers between CNT-OH tubes show that lithium ions can quickly interact with the hydroxyl groups on CNT within 2 ps. The connections of lithium ions with $-\mathrm{OH}$ groups induces the dissociations of $-\mathrm{OH}$ groups from $\mathrm{CNT}-\mathrm{OH}$ surface. More interestingly, the released hydroxyl groups can even form a water molecule, as shown in Fig. 3 (e). This process occurs within 2 ps as indicated from Fig. 3 (g).

The z-positions as a function of simulation time (ps) for each lithium ion (Fig. 3f) show that lithium ions tend to concentrate at the bottom of the CNTs, and only one ion moves into the upper part of CNT-OH. The first $\mathrm{Li}$ ion enters into the CNT-OH bundle through diffusion in $1 \mathrm{ps}$, and stays at $\mathrm{Z} \approx 0 \AA \AA$ during our 20 ps MD simulation. Five of the eight ions diffuse into the $\mathrm{CNT}-\mathrm{OH}$ interior sequentially at a time scale from 4 to 5.5 ps, and distribute in the range from $Z \approx-4 \AA$ to $Z \approx-8 \AA$. In this system, $75 \%$ lithium ions move into 
the interior of CNT-OH while 25\% stay in the interlayers between tubes as obtained from Fig. 3 (h). Previous work shows that for pristine CNT, only $37.5 \%$ lithium ions intercalate successfully while for ammonium functionalized system $87 \% .{ }^{29}$ In overall, the hydroxyl functionalized system with low lithium density and low bundle density can achieve a intercalation efficiency of $87.5 \%$. However, the intercalation of lithium ions among interlayers leads the releasing and deactivation of - $\mathrm{OH}$ groups. Therefore, how to increase the intercalation of lithium ions into interior of CNT is the key problem for hydroxyl functionalized CNT.

\section{Lithium ions intercalation into CNT bundles at high Li ion density}

Herein, an high lithium ion density of twenty lithium ions was conducted. We first simulated a hexagonal box with dimensions of $15.0 \times 15.0 \times 32.0 \AA^{3}$. As shown in Fig. 4 (a) and (b), five lithium ions diffuse into the interior of CNTs while the left ones swim in interlayers between the CNT-OH. With the increasing of free lithium ions, more hydroxyl groups dissociates and more water molecules formed (Fig. 4b). The snapshot picture and radial distribution in Fig. 4 (c) and (d), exhibiting dense arrangements of lithium ions in the CNT interior. Although the peak is not as sharp as low lithium ion density, the arrangement is relative concentrated. Lithium ions outside of the CNT sidewalls present different and asymmetric distribution. As for the radial distribution, lithium ions tend to arrange in the middle and upper sides outside of CNTs. Inside of the CNTs, lithium ions distributed relatively symmetric, with two ions locating near the upper end of CNTs as presented in Fig. 4 (d) and (e). Similar to the case of low lithium ion density with larger box, lithium ions moves into CNT-OH from the upper end. At first, seven of twenty ions vibrate and flow to the lower end and then back to the upper end, in a sequential way. The first one arrives the CNT-OH end in 1ps, as shown in the bright yellow line in Fig. 4 (f). The second ion achieves its intercalation at $\sim 4 \mathrm{ps}$, and all the other ions achieves the intercalation successively in $\sim 13$ ps and stabilize at 12-15 ps. This intercalation way is similar to the ammonium functionalized CNTs but is faster and the distribution is more concentrated. $25 \%$ of lithium ions intercalated into the interior of CNT$\mathrm{OH}$ while only $15 \%$ for ammonium functionalized $\mathrm{CNT} .{ }^{29}$ Since lithium ions act as dissociation catalysis for-OH groups, the more ions locating at the $\mathrm{CNT}-\mathrm{OH}$ interior implies less damage and dissociation to the functional groups.

High bundle density with hexagonal box dimensions of $13.0 \times 13.0 \times 32.0 \AA^{3}$ was also investigated. As depicted in Fig. 5 (a), none of the lithium ions diffuse into the interior of CNT-OH, and they all disperse randomly around CNTs interlayers instead of interior. More released hydroxyl groups or formed water molecules are observed from the side view in Fig. 5 (b). The snapshot and radial population in Fig. 5 (c) and (d) validate that none of lithium ions reach the interior space. Our examinations indicate that five of the twenty lithium ions move upwards while the left all vibrate from the bottom end into the lower or center part shown in Fig. 5 (e). Compared with low bundle density, higher bundle density will screw more lithium ions into the gaps between CNTs, resulting in the dissociation of more functional groups. These phenomena suggest that a high bundle density along with a high lithium ion concentration is unsuitable for lithium intercalation. 


\section{Energetics of lithium ions intercalation into CNT-OH}

To investigate the mechanism of intercalation, energetics of the adsorption processes were calculated to evaluate the tendency of different pathways. Before calculations, CNT-OH bundles was firstly optimized. After placing lithium ions at specified positions, the adsorption energy $E_{a d}$ is calculated as, $E_{a d}=E_{1}-E_{0}$, where $E_{1}$ denotes the energy of the system when lithium ion locates in the interior of CNTs, and $E_{0}$ representing the zero point energy of the system when lithium ions are placed at $z=11 \AA$. This is approximately $2.0 \AA$ from the top end of tubes.

The influences of different bundle densities and radial distances are depicted in Fig. 6. At the same radial distances, the bundle density affects the shapes of the $E_{a d}-Z$ curve negligibly. Careful examinations indicate that the blue curves are slightly higher than the red curves, suggesting a denser CNT-OH bundle gives lower adsorption energies. CNT-OH bundles in boxes sized $15.0 \times 15.0 \times 32.0 \AA^{3}$ and $13.0 \times 13.0 \times 32.0$ $\AA^{3}$ with the radius 0.0 and $1.0 \AA$ present similar outlines as pristine CNTs and ammonium functionalized CNTs. ${ }^{29}$ Two potential wells locate at $z$ equals 4.5 and $-4.5 \AA$. For the case of $r=1.0 \AA$, a third potential well appears at $\mathrm{z}=0 \AA$. The shapes of $\mathrm{r}=1.5 \AA$ are more complicated presenting zigzag curves and show additional apexes at $Z$ points of $1,1.5,-1,-1.5 \AA$ which are in accordance with above figures.

\section{Conclusions}

The effects of hydroxyl group modification on the interaction of lithium ions into CNT-OH bundle have been studied using ab initio MD simulation method. It is demonstrated that lithium ion can intercalate into the interior and interlayer of CNT-OH bundle. The effects of lithium ions density and bundle density on functional group changes have been carefully investigated and the dynamic processes have also been monitored.

Lower lithium ions density with low bundle density is efficient for the intercalation of lithium ions, with its efficiency as high as $87.5 \%$, which is higher than the reported pristine and ammounium functionalized CNTs. However, our calculations show that the Li ion can intercalate into the interlayers and the interiors. For the Li ions locating at the interlayer, they can catalyze the dissociation of hydroxyl functional group from the $\mathrm{CNT}-\mathrm{OH}$. Our results suggest that the intercalation into interiors of $\mathrm{CNT}-\mathrm{OH}$ is more preferential for energy storage. Further energetic calculations show that denser densities of CNT bundles induce more dispersed lithium ions in the CNT-OH interlayers, it is therefore suggested that eliminating the interlayer adsorption will be more applicable for rechargable LIBs.

\section{Declarations}

\section{Data availability}

All data generated or analyzed during this study are included in this published article. 


\section{Author Contributions}

Bin Zheng: methodology, investigation, writing original draft.

Huaze Dong: data processing

Jinmiao Zhu: picture processing

Yanping Wang: draft modification

\section{Conflicts of interest}

There are no conflicts to declare.

\section{Acknowledgements}

This work was supported by the National Natural Science Foundation of China (No. 21504021); Anhui Provincial Natural Science Foundation (No. 2108085MB32); the excellent Talent Foundation of Education Department of Anhui Province (gxyq2019066); the Key R\&D Program of Anhui Province (No. 202004a07020020) and 136 talent plan of Hefei Normal University; Collaborative Innovation Project of Anhui Universities (No. GXXT-2019-017); Natural Science Foundation of the Education Department of Anhui Province (No. KJ2020A0096).

\section{References}

1. Bahari, Y., Mortazavi, B., Rajabpour, A., Zhuang, X. Y. \& Rabczuk, T. Application of two-dimensional materials as anodes for rechargeable metal-ion batteries: A comprehensive perspective from density functional theory simulations. Energy Storage Materials 35, 203-282 (2021).

2. lijima, S. Helical microtubles of graphitic carbon. Nature 354, 56-58 (1991).

3. Terrones, M. Science and technology of the twenty-first century: Synthesis, properties and applications of carbon nanotubes. Annual Review of Materials Research 33, 419-501(2003).

4. Chang, S. L. et al. Intrinsically flexible $\mathrm{CNT}-\mathrm{TiO}_{2}$-Interlaced film for $\mathrm{NO}$ sensing at room temperature. Applied Surface Science 579 (2022).

5. Hsu, H. L. et al. Environmental-friendly three-dimensional carbon nanotubes grown by soil clay and graphene oxide nanosheets for energy storage. Materials Today Chemistry 23 (2022).

6. Kaempgen, M., Chan, C. K., Ma, J., Cui, Y. \& Gruner, G. Printable Thin Film Supercapacitors Using Single-Walled Carbon Nanotubes. Nano Letters 9, 1872-1876 (2009).

7. Park, S. K., Mahmood, Q. \& Park, H. S. Surface functional groups of carbon nanotubes to manipulate capacitive behaviors. Nanoscale 5, 12304-12309 (2013).

8. Tsang, S. C., Harris, P. J. F. \& Green, M. L. H. Thinning and opening of carbon nanotubes by oxidation using carbon-dioxide. Nature 362, 520-522, (1993). 
9. Yang, K., Gu, M. Y., Guo, Y. P., Pan, X. F. \& Mu, G. H. Effects of carbon nanotube functionalization on the mechanical and thermal properties of epoxy composites. Carbon 47, 1723-1737 (2009).

10. Chang, W. C. et al. Enhancing performance of ZnO dye-sensitized solar cells by incorporation of multiwalled carbon nanotubes. Nanoscale Research Letters 7, 1-7 (2012).

11. Lv, T., Yao, Y., Li, N. \& Chen, T. Highly Stretchable Supercapacitors Based on Aligned Carbon Nanotube/Molybdenum Disulfide Composites. Angewandte Chemie-International Edition 55, 91919195 (2016).

12. Zhao, W. Q. et al. Double polymer sheathed carbon nanotube supercapacitors show enhanced cycling stability. Nanoscale 8, 626-633 (2016).

13. Ahmad, S., Copic, D., George, C. \& De Volder, M. Hierarchical Assemblies of Carbon Nanotubes for Ultraflexible Li-Ion Batteries. Advanced Materials 28, 6705 (2016).

14. Schauerman, C. M. et al. Recycling single-wall carbon nanotube anodes from lithium ion batteries. Journal of Materials Chemistry 22, 12008-12015 (2012).

15. Zhang, D., Zhou, Y., Liu, C. H. \& Fan, S. S. The effect of the carbon nanotube buffer layer on the performance of a Li metal battery. Nanoscale 8, 11161-11167 (2016).

16. Frackowiak, E. \& Beguin, F. Electrochemical storage of energy in carbon nanotubes and nanostructured carbons. Carbon 40, 1775-1787 (2002).

17. Dileo, R. A. et al. Enhanced Capacity and Rate Capability of Carbon Nanotube Based Anodes with Titanium Contacts for Lithium Ion Batteries. Acs Nano 4, 6121-6131 (2010).

18. Korsun, O. M., Kalugin, O. N., Vasenko, A. S. \& Prezhdo, O. V. Electronic Properties of Carbon Nanotubes Intercalated with $\mathrm{Li}^{+}$and $\mathrm{Mg}^{2+}$ : Effects of Ion Charge and Ion Solvation. Journal of Physical Chemistry C 120, 26514-26521 (2016).

19. Yang, Z. H. \& Wu, H. Q. Electrochemical intercalation of lithium into carbon nanotubes. Solid State Ionics 143, 173-180 (2001).

20. Zhao, J., Buldum, A., Han, J. \& Lu, J. P. First-principles study of Li-intercalated carbon nanotube ropes. Physical Review Letters 85, 1706-1709 (2000).

21. Khantha, M., Cordero, N. A., Alonso, J. A., Cawkwell, M. \& Girifalco, L. A. Interaction and concerted diffusion of lithium in a $(5,5)$ carbon nanotube. Physical Review $B 78$ (2008).

22. Kawasaki, S., Hara, T., Iwai, Y. \& Suzuki, Y. Metallic and semiconducting single-walled carbon nanotubes as the anode material of Li ion secondary battery. Materials Letters 62, 2917-2920 (2008).

23. Ramos-Sanchez, G., Chen, G., Harutyunyan, A. R. \& Balbuena, P. B. Theoretical and experimental investigations of the Li storage capacity in single-walled carbon nanotube bundles. RSC Advances 6, 27260-27266 (2016).

24. Jia, W. B. et al. Synthesis of two novel indolo 3,2-b carbazole derivatives with aggregation- enhanced emission property. Journal of Materials Chemistry C 1, 7092-7101 (2013).

25. Gao, B. et al. Enhanced saturation lithium composition in ball-milled single-walled carbon nanotubes. Chemical Physics Letters 327, 69-75 (2000). 
26. Sozykin, S. A. \& Beskachko, V. P. Characteristics of lithium adsorption near divacancy defects on carbon nanotubes (7,7). Diamond and Related Materials 79, 127-132 (2017).

27. Jalili, S., Jamali, M. \& Schofield, J. Ab initio study of Li adsorption in carbon nanotubes functionalized with amine and carboxyl groups. Chemical Physics 418, 35-41 (2013).

28. Milowska, K. Z. \& Majewski, J. A. Functionalization of carbon nanotubes with -CHn, -NHn fragments, $\mathrm{COOH}$ and $-\mathrm{OH}$ groups. Journal of Chemical Physics 138, (2013).

29. Xiao, S. Y., Zhu, H., Wang, L., Chen, L. P. \& Liang, H. J. Enhancing the efficiency of lithium intercalation in carbon nanotube bundles using surface functional groups. Physical Chemistry Chemical Physics 16, 16003-16012 (2014).

30. Car, R. \& Parrinello, M. Unified approach for molecular-dynamics and density-functional theory. Physical Review Letters 55, 2471-2474 (1985).

31. Humphrey, W., Dalke, A. \& Schulten, K. VMD: Visual molecular dynamics. Journal of Molecular Graphics 14, 33-38 (1996).

32. Perdew, J. P., Burke, K. \& Ernzerhof, M. Generalized gradient approximation made simple. Physical Review Letters 77, 3865-3868 (1996).

33. Yingkai, Z., Weitao, Y., Perdew, J. P., Burke, K. \& Ernzerhof, M. Comment on IdquoGeneralized gradient approximation made simplerdquo and reply. Physical Review Letters 80, 890-891 (1998).

34. Troullier, N. \& Martins, J. L. Efficient pseudopotentials for plane-wave calculations. Physical Review $B$ 43, 1993-2006 (1991).

35. Kleinman, L. \& Bylander, D. M. Efficacious form for model pseudopotentials. Physical Review Letters 48, 1425-1428 (1982).

36. Billeter, S. R., Curioni, A. \& Andreoni, W. Efficient linear scaling geometry optimization and transitionstate search for direct wavefunction optimization schemes in density functional theory using a plane-wave basis. Computational Materials Science 27, 437-445 (2003).

37. Nose, S. A unified formulation of the constant temperature molecular-dynamics methods. Journal of Chemical Physics 81, 511-519 (1984).

38. Hoover, W. G. Canonical dynamics: Equilibrium phase-space distributions. Physical Review A 31, 1695-1697 (1985).

\section{Figures}

\section{Figure 1}

Initial conformation of the unit cell for ab initio MD simulations. The lithium and hydrogen atoms are shown in orange and light gray, respectively; the carbon atoms are displayed in dark gray; and the oxygen atoms are in red. (a) and (b) are the - $\mathrm{OH}$ functionalized CNT with four and ten lithium atoms placed upon 
each end of the CNT, respectively. (a) and (b): top view; (c): side view. The symbol 0 indicates the origin of the coordinates. The radial distance between the lithium atoms from the central axis of the nanotube is denoted by the symbol r used together with the black arrow.

(a)

(c)
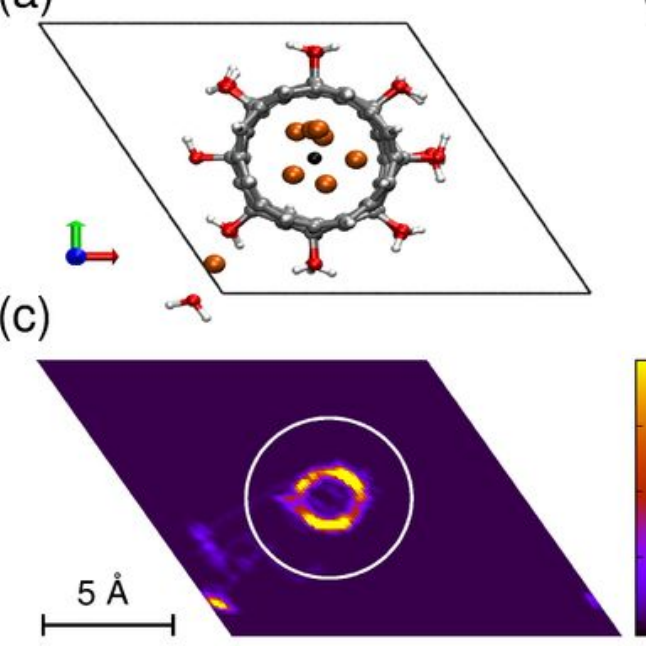

(d)

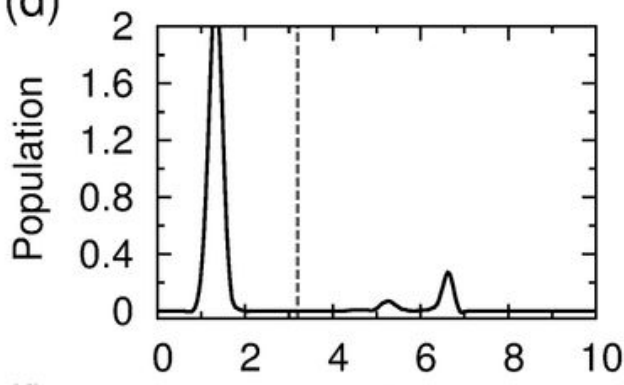

(f)

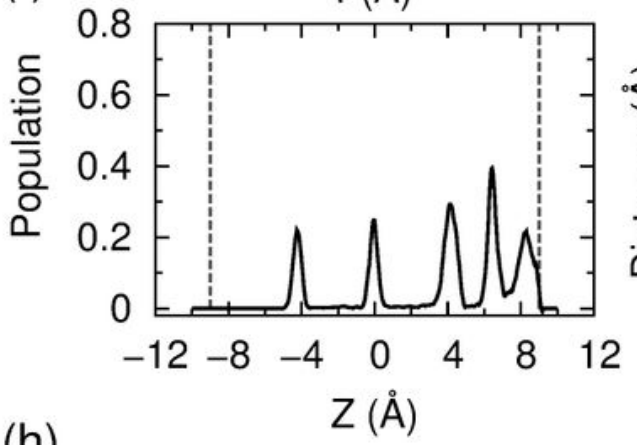

(h)

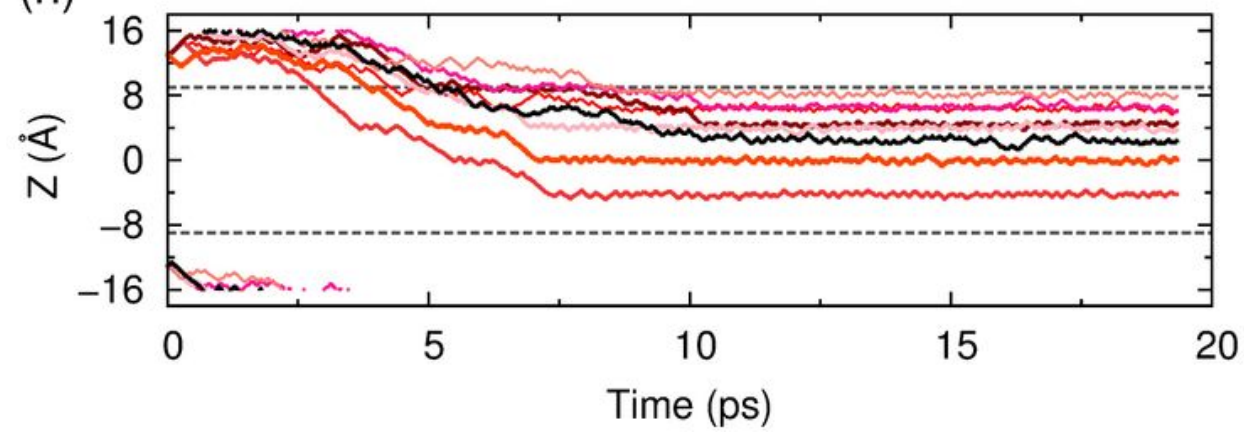

(b)

(e)

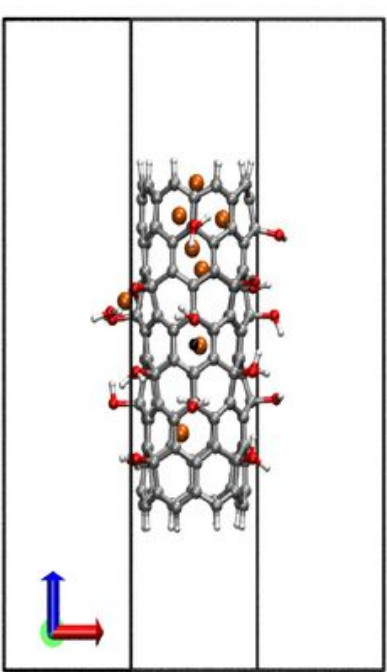

(g)

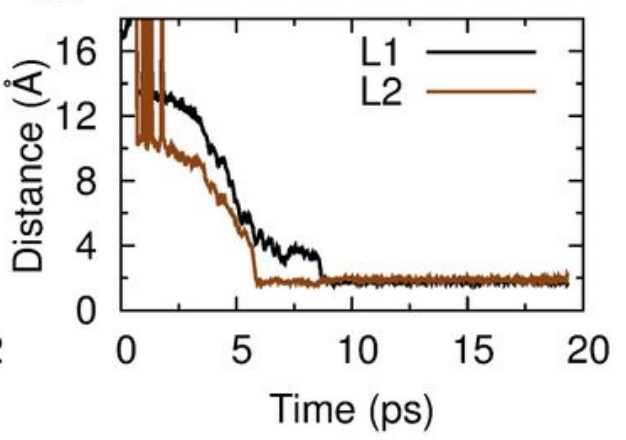

Figure 2 
Behavior of the eight $\mathrm{Li}$ ions intercalating and diffusing into the bundle of $-\mathrm{OH}$ functionalized CNTs. The system was simulated in a hexagonal box with dimensions $15.0 \times 15.0 \times 32.0 \AA^{3}$.(a) Typical snapshot of the unit cell, view from the top. (b) side view. (c) Distribution of the Li ions in the x-y plane. (d) Radial distribution of the Li ions, and the vertical dashed line represents the wall of nanotube. (f) Axial distribution of the ions within CNT, only calculated for the ions within the nanotube. The two ends of CNT are indicated by the two vertical dashed lines. (e) a snapshot for structure of the $-\mathrm{OH}$ groups and the $\mathrm{Li}$ ion located at the space between the nanotubes, and the distance between $\mathrm{Li}$ and oxygen atoms are displayed in (g). (h) The z-position as a function of simulation time (ps) for each lithium ion. The upper and lower dashed gray horizontal lines denote the top and bottom ends of CNT, respectively. 
(a)

(c)
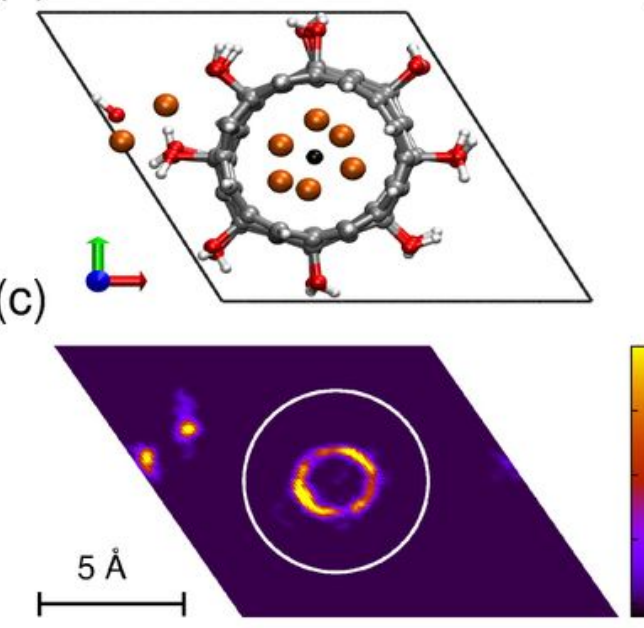

(d)

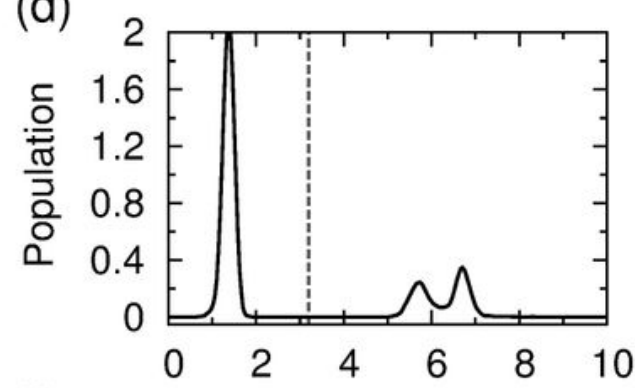

(f)

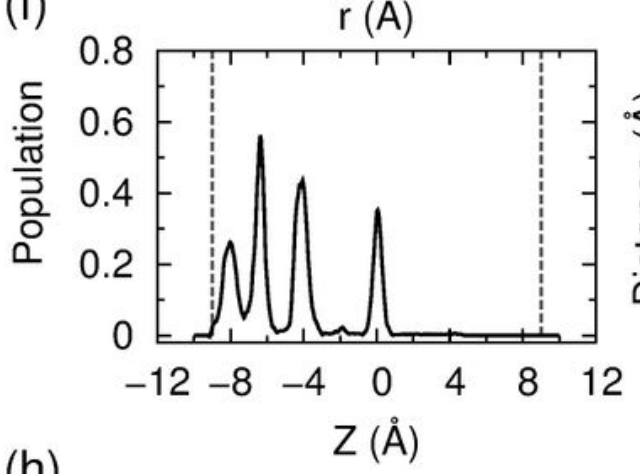

(b)

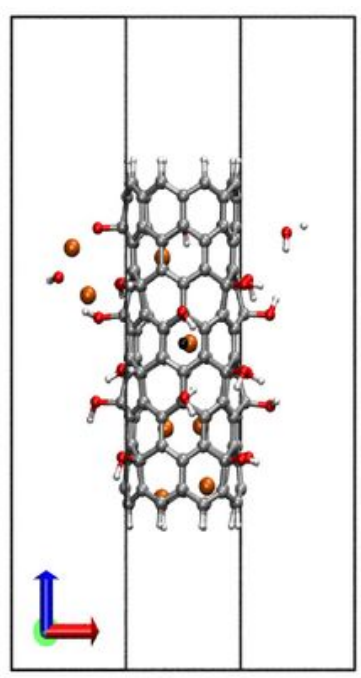

(e)

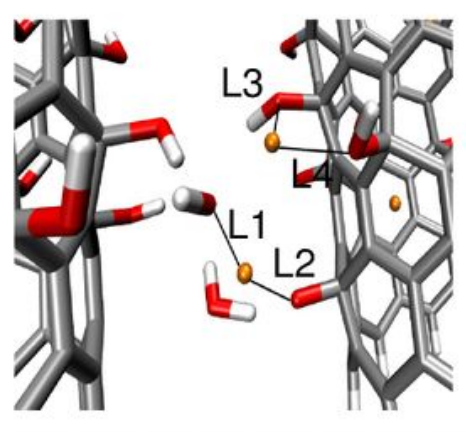

\section{(g)}
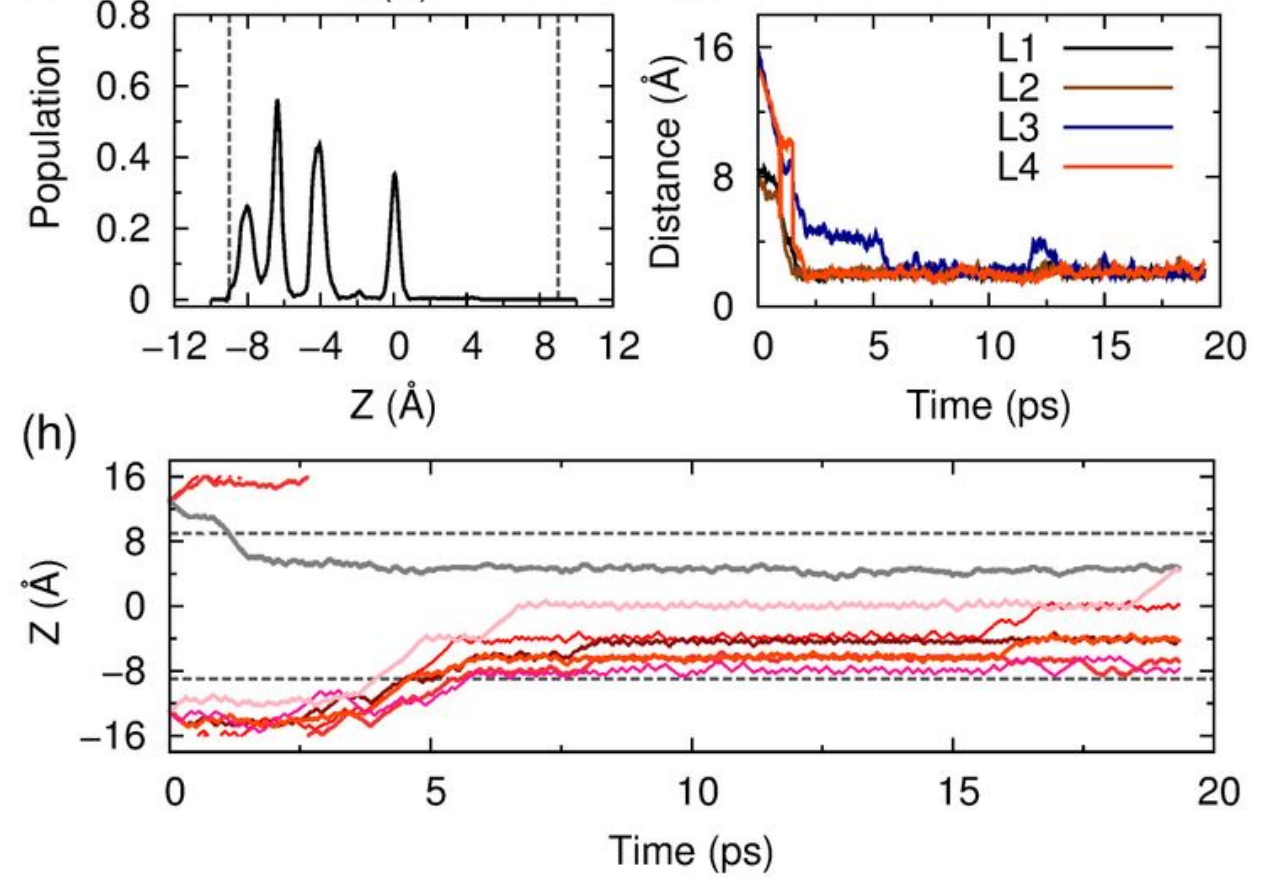

\section{Figure 3}

Behavior of the eight $\mathrm{Li}$ ions intercalating and diffusing into the bundle of $-\mathrm{OH}$ functionalized CNTs. The system was simulated in a hexagonal box with dimensions $13.0 \times 13.0 \times 32.0 \AA^{3}$. (a) Typical snapshot of the unit cell, top view. (b) Side view. (c) Distribution of the Li ions in the x-y plane. (d) Radial distribution of the Li ions,and the vertical dashed line represents the wall of nanotube. (f) Axial distribution of the ions within CNT, only calculated for the ions within the nanotube. The two ends of CNT are indicated by the 
two vertical dashed lines. (e) A snapshot for structure of the -OH groups and the $\mathrm{Li}$ ion located at the space between the nanotubes, and the distance between Li and oxygen atoms are displayed in $(\mathrm{g}) .(\mathrm{h})$ The z-position as a function of simulation time (ps) for each lithium ion. The upper and lower dashed gray horizontal lines denote the top and bottom ends of CNT, respectively. Because the simulation is performed under periodic boundary condition, these ions that move down towards the bottom edge of the simulation box will simultaneously appear at the top edge of the box.

(a)

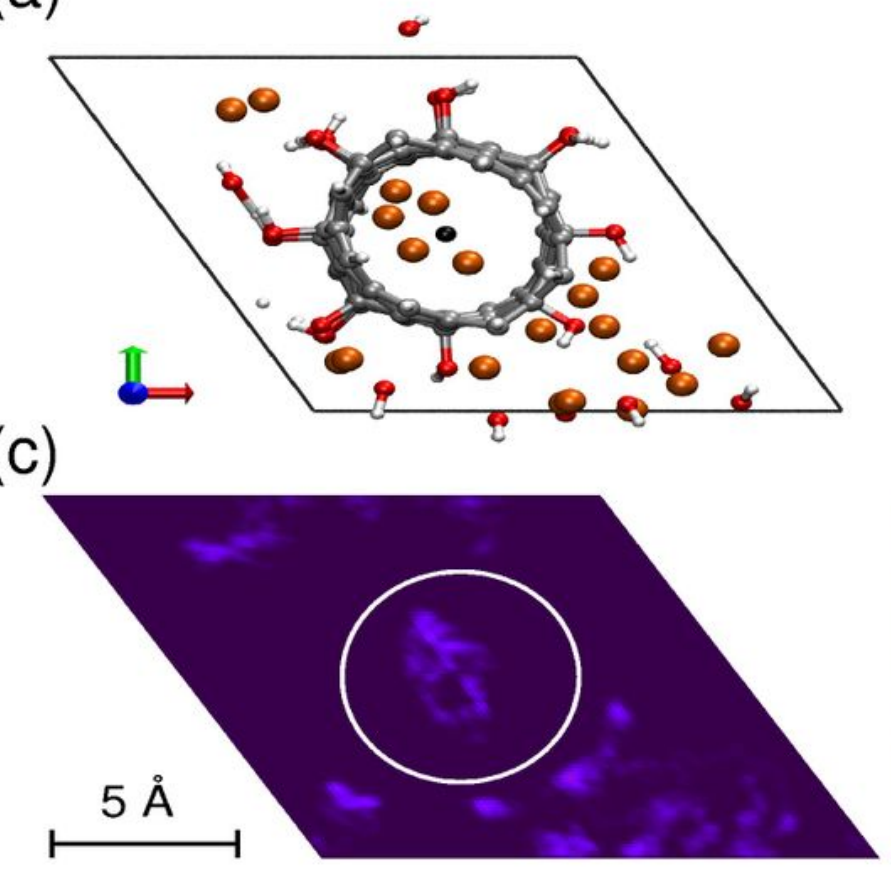

(d)

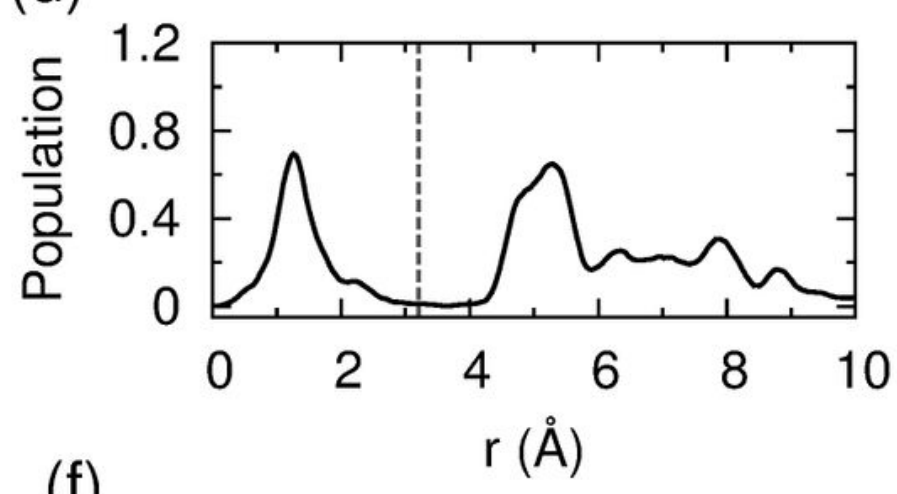

(b)

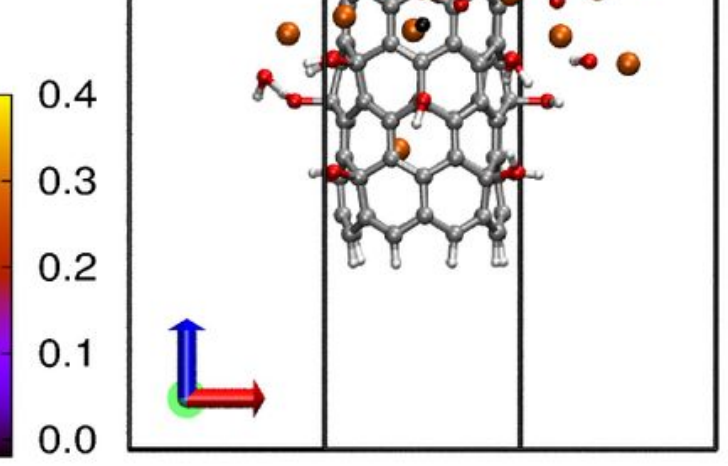

(e)

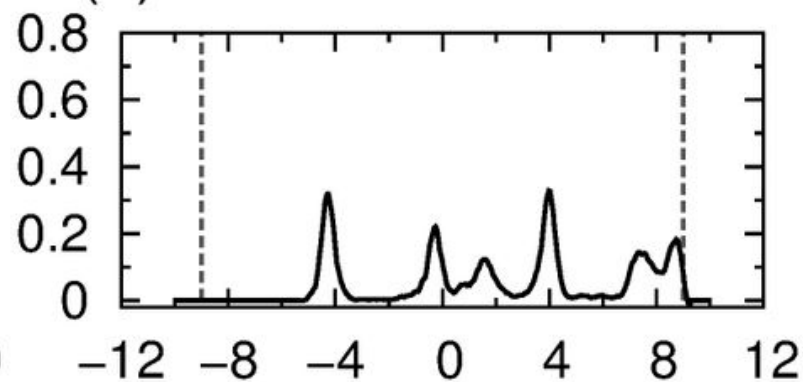

$Z(\AA)$

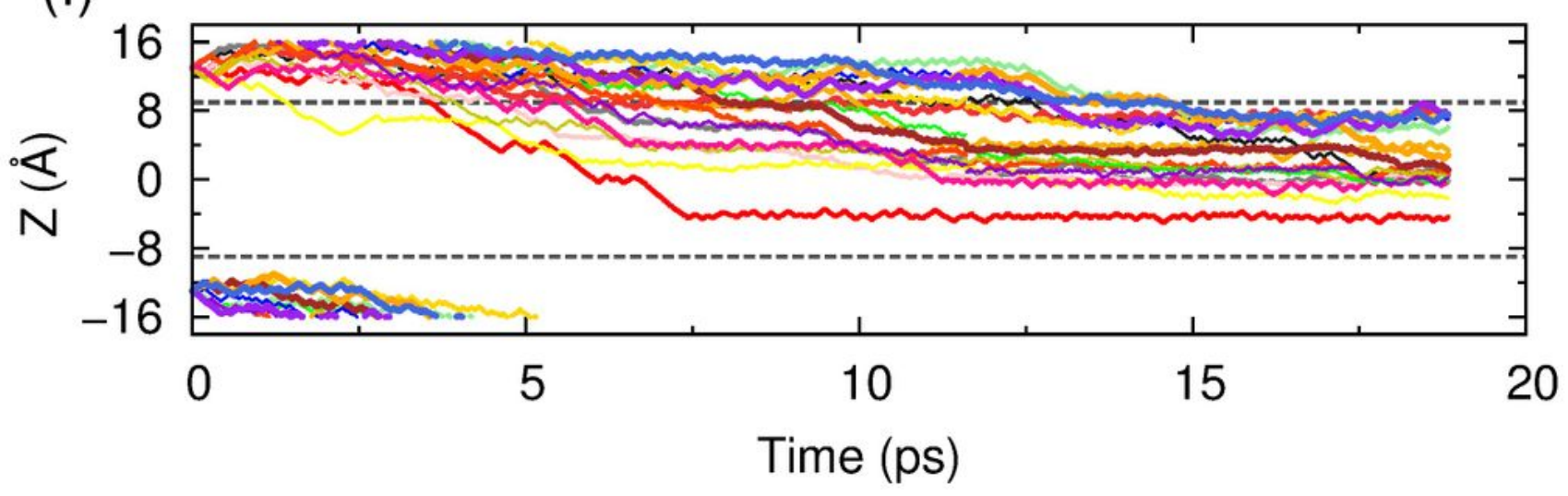


Figure 4

Behavior of the twenty lithium ions intercalating and diffusing into the bundle of the -OH functionalized CNTs, simulated in a hexagonal box with dimensions of $15.0 \times 15.0 \times 32.0 \AA^{3}$. (a) Typical snapshot of the unit cell, top view. (b) side view. (c) Distribution of the lithium ions in the x-y plane. (d) Radial distribution of the Li ions. (e) Axial distribution of the ions within CNT. (f) The z-position as a function of simulation time (ps) for each lithium ion.

\section{Figure 5}

Behavior of the twenty lithium ions intercalating and diffusing inside or between $-\mathrm{OH}$ functionalized CNTs. The system was simulated in a hexagonal box with dimensions $13.0 \times 13.0 \times 32.0 \AA^{3}$. (a) Typical snapshot of the unit cell, view from the top. (b) Side view. (c) Distribution of the lithium ions in the $x-y$ plane. (d) Radial distribution of the lithium ions, and the vertical dashed line represents the wall of nanotube. (e) The z-position as a function of simulation time (ps) for each lithium ion. The upper and lower dashed gray horizontal lines denote the top and bottom ends of CNT, respectively.

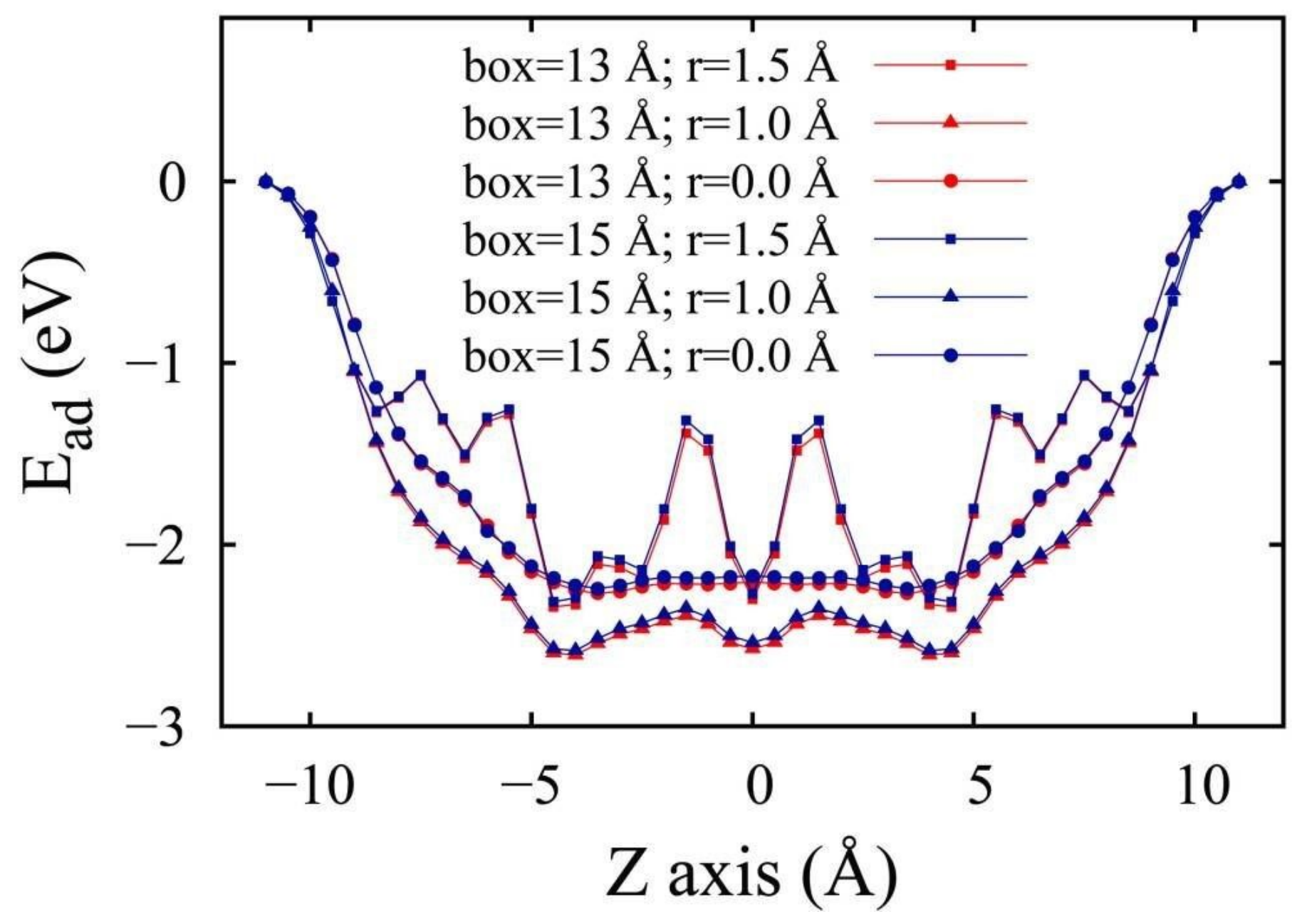


Figure 6

Adsorption energy of a lithium atom within the - $\mathrm{OH}$ functionalized CNT. The lithium atoms is placed at position $z$ and in the interior at $r=1.5 \AA, 1.0 \AA$ and $0.0 \AA$. The two ends of the nanotube are indicated by the vertical dashed lines. 